\title{
Glauber dynamics and ageing
}

\author{
R. Mélin ${ }^{* \dagger}$ and P.Butaud ${ }^{\ddagger}$ \\ CRTBT-CNRS, 25 Avenue des Martyrs, BP 166X, 38042 Grenoble CEDEX, France
}

September 6, 2018

\begin{abstract}
The Glauber dynamics of various models (REM-like trap models, Brownian motion, BM model, Ising chain and SK model) is analyzed in relation with the existence of ageing. From a finite size Glauber matrix, we calculate a time $\tau_{w}(N)$ after which the system has relaxed to the equilibrium state. The case of metastability is also discussed. If the only non zero overlaps between pure states are only self-overlaps (REM-like trap models, BM model), the existence or absence of ageing depends only on the behavior of the density of eigenvalues for small eigenvalues. We have carried out a detailed numerical and analytical analysis of the density of eigenvalues of the REM-like trap models. In this case, we show that the behavior of the density of eigenvalues for typical trap realizations is related to the spectral dimension of the equivalent random walk model.
\end{abstract}

\footnotetext{
*e-mail: melin@crtbt.polycnrs-gre.fr

${ }^{\dagger}$ Present address: International School for Advanced Studies (SISSA), Via Beirut 2-4, 34014 Trieste, Italy

${ }^{\ddagger}$ e-mail: butaud@crtbt.polycnrs-gre.fr
} 


\section{Introduction}

Various approaches have been used to understand ageing experiments [1] [2] [3]: droplet picture [4], mean field model [5] [6], and trap models [7] [8] [9] [10]. Ageing is by definition the property that the two points correlation function

$$
C\left(t_{w}, t\right)=\left\langle\mathcal{O}\left(t_{w}+t\right) \mathcal{O}\left(t_{w}\right)\right\rangle
$$

depends explicitly on the waiting time $t_{w} . \mathcal{O}$ is a macroscopic observable, for instance the magnetization in the zero field cooled experiment [1] or the thermoremanent magnetization experiment [2]. On the theoretical level, dynamics can be implemented in several ways. One possibility is Langevin dynamics which was used for instance in [11] where mean field ageing was first obtained, or in [12] where ageing in the presence of flat directions was studied. Another possibility is Glauber dynamics [13]. Within the framework of Glauber dynamics and given the probability distribution $p\left(\left\{e_{i}\right\}, 0\right)$, for the system to be in any microcanonical state $e_{i}$ at time $t=0$, the probability distribution at time $t$ is given by

$$
p\left(\left\{e_{i}\right\}, t\right)=\exp (T t) p\left(\left\{e_{i}\right\}, 0\right),
$$

where $T$ is the Glauber matrix. By "microcanonical states", we mean a state such as the system is in a given microcanonical configuration with probability one.

This article is devoted to the study of the Glauber dynamics of various models, phenomenological or microscopic, in connection with ageing. More precisely, for a finite size system of size $N$, we calculate from the Glauber matrix a time $\tau_{w}(N)$ such as the finite size system equilibrates on a time of the order of $\tau_{w}(N)$, starting from out-of-equilibrium initial conditions at time $t=0$. In terms of ageing, the correlation function (11) depends explicitely on the waiting time $t_{w}$ provided the system is out-of-equilibrium at time $t_{w}$, namely $t_{w}$ is smaller than $\tau_{w}(N)$. In other words, ageing for a finite size system is interupted after $\tau_{w}(N)$ [see [7] where the denomination "interrupted ageing" was proposed]. In order to understand ageing in the thermodynamic limit $(N \rightarrow \infty)$, the variations of $\tau_{w}(N)$ with the system size have to be carefully studied. If $\tau_{w}(N)$ goes to a constant in the thermodynamic limit, ageing is interrupted, whereas if $\tau_{w}(N)$ diverges ageing is not interrupted in the thermodynamic limit: in the thermodynamic limit, the system reaches equilibrium on infinite time scales. We also discuss the possibility of metastability without ageing on experimental time scales, as it is the case for instance in diamond.

In general, $\tau_{w}(N)$ depends on the eigenvalues of the Glauber matrix, but also on the overlaps between microcanonical states, and the transformation matrix elements between the eigenstates of the Glauber matrix and the microcanonical states. However, in some simple cases, with only selfoverlaping states, the time $\tau_{w}(N)$ can be expressed only in terms of the density of eigenvalues of the Glauber matrix. 
The form of the Glauber matrix will be given in each case in the core of the paper. Whatever the system, the eigenvalues of $T$ are negative. The spectrum is bounded above by zero (zero is always an eigenvalue, corresponding to the equilibrium Boltzmann distribution). The eigenvalues of the Glauber matrix have the dimension of the inverse of a time, so that they can be viewed as the inverse relaxation times of the system. Since we are interested in long time behavior, a special attention will be paid to the smallest eigenvalues.

This article is organized as follows. In section 2, we present a detailed study of the average density of small eigenvalues of the random energy model (REM) like trap model proposed and studied in [7, 8, 9] and inspired by the REM model [14].

In the case of the REM-like trap model, two regimes are predicted and checked numerically for the density of small eigenvalues in the glass phase: an Arrhenius-like regime and a non Arrhenius regime for the smallest eigenvalues. By Arrhenius-like regime, we mean a regime in which the density of eigenvalues is the same as the density of eigenvalues calculated from the Arrhenius law, assuming a correspondence between the eigenvalues of the Glauber matrix and the inverse relaxation times of the system. The eigenvalue density in the Arrhenius regime are given in section 2.1. We also establish a connection between the behavior of the density of small eigenvalues of REM-like trap models and the spectral dimension of the associated diffusion process.

Next, in section 3, we give a general relation for the existence of ageing from a Glauber dynamics point of view. This section is the main part of the present article. When applied to the REM-like trap model, our criterium is equivalent to the fact noticed in [7] that the average trapping time diverges below $T_{g}$. The rest of the paper is devoted to examine the case of other models: Brownian motion which was shown in [12] to be an ageing phenomenon, a model introduced by Barrat and Mézard [15] (BM model), the one dimensional Ising chain (in this case, our results are consistent with the existence of interrupted ageing), and the Sherrington-Kirkpatrick model (SK model). In all these cases, we find consistent results between the existence of ageing calculated from the behavior of the two times autocorrelation functions and our criterium based on the Glauber matrix. We end-up with some final remarks.

\section{Density of small eigenvalues of trap models (REM-like trap mod- els and trap models with other topologies)}

Trap models can be defined without a priori reference to a microscopic model. However, some trap models, such as the REM-like trap model [9] are related to the Parisi solution of spin glasses: even though these models are toy models, some of them retain some feature of the dynamics of microscopic models. The REM-like trap model consists of a trap model in infinite dimension, that is, the system

can "hop" from one trap to any other trap, according to the rules of the dynamics. Other toplogies will be analyzed below, in reduced dimension. The REM-like trap model corresponds to a single 
replica symmetry breaking step. Generalizations of the REM-like trap model including multi replica symmetry breaking steps were studied in [9] and their relevance to explain experimental data was shown.

The models of this section consist of $N$ traps of depth $E_{i}<0$, with the $E_{i}$ chosen among the distribution $P(E)=\exp (E)$. The reason why this particular energy trap distribution is chosen is that there exists a finite transition temperature between a high and a low temperature glassy phase [10]. The dynamics rules are given by $T_{i, j} \propto \exp \left(\beta E_{j}\right)$ for $i \neq j$ if the traps $i$ and $j$ are connected and zero otherwise. The diagonal terms are defined by

$$
\sum_{k=1}^{N} T_{k, i}=0
$$

which enforces probability conservation. Equation (3) is a general constraint for any Glauber dynamics. Another constraint is the detailed balance condition

$$
T_{j, i} \exp \left(-\beta E_{i}\right)=T_{i, j} \exp \left(-\beta E_{j}\right)
$$

It is clear that the aforementioned rules for the dynamics of the REM-like trap model satisfy detailed balance. However, as we shall see below, other types of dynamics also satisfy detailed balance and probability conservation. As a consequence of detailed balance, the eigenvectors of the Glauber matrix are not physical states (in the sense that they are not probability distributions) except for the Boltzmann distribution (the stationary eigenvector corresponding to the $\lambda=0$ eigenvalue). Notice that if $T$ is a Glauber matrix $x T$, with $x$ a positive number, is also a Glauber matrix. Multiplying $T$ by $x$ amounts to changing the unit time. The proportionality constant is imposed by physical considerations. For the models considered in this section, this constant is chosen such as the diagonal elements of the Glauber matrix do not scale with the number $N$ of traps in the large $N$ limit. For instance, in the infinite dimensional case, we choose $T_{i, j}=\exp \left(\beta E_{j}\right) / N$. This dynamics will be studied with different phase space topologies: infinite dimension, where all the traps are connected (section 2.2), one dimensional topology, where a given trap is connected only to its two nearest neighbors (section 2.3), and also one dimensional topology with "ordered" traps, in the sense that the trap energies have been ordered $E_{1}<\ldots<E_{N}$, and the trap at site $i$ has an energy $E_{i}$.

A useful property that we will use throughout this paper is that Glauber matrices can be mapped onto symmetric matrices with the same spectrum. Let us call $V$ the diagonal matrix $V_{i, j}=\exp \left(\beta E_{i} / 2\right) \delta_{i, j}$. Then, because of detailed balance, the matrix $\tilde{T}=V T V^{-1}$ is symmetric and has the same spectrum as $T$. Using a quantum mechanical analogy, we denote by $\left|e_{i}\right\rangle$ the microcanonical state corresponding the system in trap $i$ with a probability unity. The analogy to quantum mechanics is useful, even though there is no hermitian structure. In particular, any linear combination of microcanonical states is not a probability distribution. 


\subsection{Arrhenius regime}

We propose ourselves to carry out a detailed study of the density of eigenvalues of the REM-like trap model and related models in reduced dimension. Since the Arrhenius law is introduced "by hand" in the dynamics, it is interesting to question in which regime the density of eigenvalues is given by the Arrhenius law, assuming a correspondence $\tau=-1 / \lambda$ between the eigenvalues of the Glauber matrix and relaxation times. We will naturally call Arrhenius regime such a regime. As we will see below, the density of eigenvalues is not always in the Arrhenius regime.

In this Arrhenius regime, and by definition, the off-diagonal terms are neglectable as far as the density of eigenvalues is concerned. The diagonal coefficients of the Glauber matrix are proportional to $\exp \left(\beta E_{i}\right)$, so that the distribution of eigenvalues is

$$
P(\ln |T|) \propto \exp \left(\frac{1}{\beta} \ln |T|\right) .
$$

This distribution is the same as the one expected from an Arrhenius law with life times $\tau_{i} \propto$ $\exp \left(-\beta E_{i}\right)$. If one assumes an Arrhenius picture, the distribution of relaxation times is

$$
P(\tau) \propto \frac{1}{\tau^{1+1 / \beta}},
$$

which is equivalent to (5). As noticed in [9], the average trapping time diverges if $\beta>1$, leading to ageing. Distribution of trapping times with no first moment were already studied in [18], and shown to lead to anomalous diffusion, namely to a non trivial spectral dimension, even above the upper critical dimension. We will compare our results for the REM-like trap model with the results of [18] derived of the context of anomalous diffusion on site-disordered hypercubic lattices.

The density of eigenvalues is thus described by the Arrhenius law provided the off-diagonal terms of the Glauber matrix are a small perturbation, so that the density of eigenvalues of the Glauber matrix is given by the diagonal terms only. We now examine several cases with different phase space topologies and determine in the different cases on which condition the density of eigenvalues is determined by the Arrhenius picture. We also compare our analytical approach to numerical diagonalizations of the symmetrized matrix $\tilde{T}$.

\subsection{Infinite dimensional case: REM-like trap model}

In this section, the system can hop from one trap to any other trap (infinite dimension). The diagonal coefficients are

$$
T_{i, i}=\tilde{T}_{i, i}=-\frac{N-1}{N} \exp \left(\beta E_{i}\right),
$$

and the off-diagonal coefficients of the symmetrized Glauber matrix are

$$
\tilde{T}_{i, j}=\frac{1}{N} \exp \left(\frac{\beta}{2}\left(E_{i}+E_{j}\right)\right) .
$$




\subsubsection{Determination of the Arrhenius regime}

We treat the off-diagonal coefficients $\tilde{T}_{i, j}(i \neq j)$ as a perturbation. This perturbative treatment is consistent since we are looking for a regime in which the off-diagonal coefficients do not modify the density of eigenvalues with respect to the purely diagonal case. The second order correction to the eigenvalue $T_{i, i}$ is

$$
\delta_{i}^{(2)}=\frac{1}{2 N(N-1)} \sum_{k \neq i} \frac{\exp \left(\beta\left(E_{i}+E_{k}\right) / 2\right)}{\sinh \left(\beta\left(E_{k}-E_{i}\right) / 2\right)},
$$

the first order corrections being identically zero since the perturbation consists only of off-diagonal elements. We assume that the density of trap energies in the vicinity of $E_{i}$ is large enough for the discrete summation to be replaced by an integral. If this condition is not satisfied, the second order corrections $\delta_{i}^{(2)}$ may have large fluctuations because the divergence for $E_{k} \rightarrow E_{i}$ is no more cancelled. The condition that the unperturbed eigenvalue density is large in the vicinity of $E_{i}$ simply reads $N \exp \left(E_{i}\right) \gg 1$. We assume in the rest of this section that this condition is fulfilled, and we can safely replace the discrete sum by an integral:

$$
\delta_{i}^{(2)}=\frac{1}{2(N-1)} \int_{-\infty}^{0} \frac{\exp \left(\beta\left(E_{i}+E\right) / 2\right)}{\sinh \left(\beta\left(E-E_{i}\right) / 2\right)} e^{E} d E .
$$

The singularities for $E=E_{i}^{+}$and $E=E_{i}^{-}$cancel each other, and we can approximate $\delta_{i}^{(2)}$ as

$$
\delta_{2}^{(i)} \simeq \frac{1}{N-1} B \exp \left((1+\beta) E_{i}\right)
$$

with $B=4(1+\beta / 2) / \beta^{2}$. We have used the following approximation: if $A$ is small, then

$$
\int_{-A}^{A} \frac{\exp (\lambda x)}{\sinh (\mu x)} \simeq \frac{2 \lambda A}{\mu}
$$

and we use $A=2 / \beta$ as a cut-off. Clearly (11) is only an approximation, but we are only interested in the dominant behavior of $\delta_{i}^{(2)}$ as a function of $E_{i}$. Now, we want to compare the approximation (11) of $\delta_{i}^{(2)}$ to the typical level spacing between $T_{i, i}$ and $T_{i+1, i+1}$, where we assume that the traps are ordered such as $E_{1}<E_{2}<\ldots<E_{N}$. To to so, we first calculate the distribution of

$$
|T|=\frac{N-1}{N} \exp (\beta E),
$$

and we obtain $P(|T|)=A|T|^{1 / \beta-1}$, with

$$
A=\frac{1}{\beta}\left(\frac{N}{N-1}\right)^{1 / \beta} .
$$

Notice here that $P(|T|)$ diverges as $|T|$ goes to zero if $\beta>1$. This accumulation of small relaxation times is related to the existence a glass transition for this model for $\beta=1$. Let us now calculate the level spacing statistics $P_{|T|}(S)$, which is the density probability that the spacing between the 
absolute value of the eigenvalue $T$ and the absolute value of the next eigenvalue is $S$. Because of the statistical independence of the trap depths, $P_{|T|}(S)$ is readily obtained as

$$
P_{|T|}(S) \delta S=\sum_{k=1}^{N-1}\left(\begin{array}{c}
N-1 \\
k
\end{array}\right)(\delta S P(|T|+S))^{k}\left(\int_{0}^{|T|}+\int_{|T|+S+\delta S}^{1} P(t) d t\right)^{N-k-1} .
$$

In this expression, $k$ is the number of levels in the interval $[|T|+S,|T|+S+\delta S]$ (with $k \geq 1$ ). The other terms correspond to the condition that there is no eigenvalue in the interval $[|T|,|T|+S]$. Assuming that $|T|$ is small compared to unity, and that $N$ is large, we get

$$
P_{|T|}(S) \simeq \frac{1}{\beta} N(|T|+S)^{1 / \beta-1} .
$$

The off-diagonal terms are irrelevant to the density of eigenvalues provided

$$
\mathcal{P}(|T|)=\int_{0}^{\delta_{i}^{(2)}} P_{|T|}(S) d S
$$

is much smaller than unity. This condition reads $B e^{2 E_{i}} / \beta \ll 1$, with $B$ defined in (11). Since we are looking for a necessary condition valid if $\beta>1$, we neglect the temperature-dependent prefactor and conclude that the density of states is in the Arrhenius regime only for traps such that $1 / N \ll e^{E} \ll 1$. Notice that the traps such as $N \exp E \ll 1$ are deeper than the typical deepest trap (of the order of $-\ln N)$. The Arrhenius picture is thus valid for a typical trap realization, with a lowest trap not deeper than $-\ln N$. However, non typical disorder realizations may be generated. For these realizations, the lowest eigenvalues are not described by an Arrhenius picture. The existence or absence of the Arrhenius regime even for a very large number of traps is thus sample dependent. This non self averaging behavior is reminiscent on the sample dependence of the quantity

$$
Y=\sum_{i=1}^{N} W_{i}^{2}
$$

with $W_{i}$ the probability to find the system in trap $i$ [17].

\subsubsection{Numerical tests}

The density of small eigenvalues of the REM-like trap model is pictured on figure 1. For practical purposes, we do not calculate the density $P(\ln |\lambda|)$ since this quantity is too small for small eigenvalues (see (5)). We rather calculate $P_{B}(\ln |\lambda|)=W_{B} P(\ln |\lambda|)$, where $W_{B}$ is the Boltzmann weight associated to the eigenvalue $\lambda$, which is calculated as the average of the Boltzmann weight operator

$$
\hat{W}_{B}=\sum_{i=1}^{N}\left|e_{i}\right\rangle e^{-\beta E_{i}}\left\langle e_{i}\right|
$$

over the eigenstate associated to the eigenvalue $\lambda$. In the Arrhenius picture, we have

$$
P_{B}(\ln |\lambda|) \propto \exp \left(\left(\frac{1}{\beta}-1\right) \ln |\lambda|\right) .
$$


Since $\beta>1, P_{B}(\ln |\lambda|)$ increases exponentially as a function of $\ln |\lambda|$ when $\lambda \rightarrow 0^{-}$. We see on figure 10 the existence of the Arrhenius regime, in good agreement with the condition $1 / N \ll e^{E} \ll 1$. We can now fit the density of eigenvalues in the Arrhenius regime to the form

$$
P_{B}(\ln |\lambda|) \propto \exp (-\alpha \ln |\lambda|)
$$

and see if we recover $\alpha=1-T$, as predicted from the Arrhenius law. The fit to the Arrhenius law is shown on figure 2. The variations of the exponent $\alpha$ as a function of the inverse temperature are plotted on figure 3, and we conclude to a good agreement with the Arrhenius law.

\subsubsection{Relation with the anomalous diffusion on site-disordered lattices}

Random walks on a finite $d$-dimensional lattice with a broad distribution of waiting times have been analyzed in the past (see [18] and references therein). As we shall see, we can relate our results concerning the existence of a Arrhenius-like regime for the REM-like trap model to the results of [18], where the long time behavior of random walk on such lattices was investigated by means of the real space renormalization method introduced in [19]. The REM-like trap model falls in the universality class described by Machta since the ingredients are the same in the two cases, rather than the universality class of random walks in a random field force [20]. In order to make contact with the work of Machta, we first calculate the probability $P_{0}^{(R E M)}(t)$ of being at the origin:

$$
P_{0}^{(R E M)}(t)=\overline{\frac{1}{N} \sum_{i=1}^{N} \mathcal{P}\left(e_{i}, t \mid e_{i}, 0\right)}=\overline{\frac{1}{N} \sum_{\alpha} e^{\lambda_{\alpha} t}},
$$

where $\mathcal{P}\left(e_{i}, t \mid e_{i}, 0\right)$ is the probability to find the system in the trap $i$ at time $t$, starting from the trap $i$ at time 0 , and $\left\{\lambda_{\alpha}\right\}$ is the set of eigenvalues of the Glauber matrix. We have shown that, in the infinite dimensional case, the typical small eigenvalue density (relevant to the long time behavior of $\left.P_{0}(t)\right)$ has the Arrhenius form $\rho(\lambda) \sim \lambda^{T-1}$. A simple scaling analyzis then shows that

$$
P_{0}^{(R E M)}(t) \sim t^{-T}
$$

in the long time limit. This result was derived for a graph such as any site is connected with any other site, that is we started from the begining from an infinite dimensional situation. In order to extrapolate this result to a finite dimension $d$ larger than the upper critical dimension 2 (as shown in [18]) we first notice that, within the framework of the REM-like trap model, the microscopic time $\tau_{0}$ scales like $\tau_{0}^{(R E M)} \sim a^{d}$, with a the lattice spacing, whereas, in the context of random walks, the correct scaling is $\tau_{0}^{(R W)} \sim a^{2}$ [an illustration of the validity of such a scaling will be given in section $\llbracket$. This implies that $\tau_{0}^{(R W)} \sim\left(\tau_{0}^{(R E M)}\right)^{2 / d}$, which gives the correct prescription to go from the REM-like trap model in infinite dimension to the finite $d$ dimensional random walk model: one should replace the time $t$ in $(23)$ by $t^{d / 2}$. This leads to

$$
P_{0}^{(R W)} \sim t^{-T d / 2}
$$


for $d$ larger than the upper critical dimension 2. This is exactly what was found in [18] using a real space renormalization group approach, with a non trivial spectral dimension $d_{s}=T d$.

\subsubsection{Structure of the eigenvectors of the REM-like trap model}

We aim to calculate the structure of the eigenstates of the REM-like trap model. To do so, we decompose a given microcanonical state $\left|e_{i}\right\rangle$ into the eigenvectors $\left|\psi_{j}\right\rangle$, and calculate the absolute value of the coefficients of this decomposition. The result is plotted on figure $₫$. We see that a microcanonical state $\left|e_{i}\right\rangle$ with an eigenvalue $\lambda_{i}$ is mainly a linear combination of eigenstates with eigenvalues close to $\lambda_{i}$. This observation can be understood in the framework of perturbation theory as follows. The first order correction to the eigenvectors are

$$
\left|\delta \phi_{i}\right\rangle=\frac{1}{2(N-1)} \sum_{k \neq i} \frac{1}{\sinh \left(\beta\left(E_{k}-E_{i}\right) / 2\right)}\left|e_{k}\right\rangle,
$$

so that, at first order

$$
\left|e_{i}\right\rangle=\left|\psi_{i}\right\rangle-\frac{1}{2(N-1)} \sum_{k \neq i} \frac{1}{\sinh \left(\beta\left(E_{k}-E_{i}\right) / 2\right)}\left|\psi_{k}\right\rangle .
$$

At the first order of perturbation theory, the pure states $\left|e_{i}\right\rangle$ is thus mainly a linear combination of eigenvectors with an eigenvalue close to the eigenvalue of $\left|\psi_{i}\right\rangle$.

Starting from a pure state $\left|e_{i}\right\rangle$ at time $t=0$, we can calculate the evolution of the system:

$$
\begin{aligned}
\left|e_{i}(t)\right\rangle= & \exp \left(-\frac{N-1}{N} e^{\beta E_{i}} t\right)\left|e_{i}\right\rangle \\
& +\frac{1}{2(N-1)} \sum_{k \neq i} \frac{1}{\sinh \left(\beta\left(E_{k}-E_{i}\right) / 2\right)}\left[\exp \left(-\frac{N-1}{N} e^{\beta E_{i}}\right)-\exp \left(-\frac{N-1}{N} e^{\beta E_{k}} t\right)\right]
\end{aligned}
$$

where we have used (25) and (26). As expected from the Arrhenius law, the relaxation time is of the order of $\tau_{i}=\exp \left(-\beta E_{i}\right)$, which diverges in the limit $\beta \rightarrow+\infty$. We will compare below the structure of the eigenvectors of the REM-like trap model to the structure of eigenvectors of another model (BM model).

\subsection{One dimensional case with disordered traps}

\subsubsection{Absence of Arrhrenius regime}

Assuming periodic boundary conditions, the diagonal terms of the Glauber matrix are given by

$$
T_{i, i}=-2 \exp \left(\beta E_{i}\right)
$$

The perturbation induced by the off-diagonal terms does not affect significatively the density of eigenvalues provided the perturbation $\tilde{T}_{i, j}=\exp \left(\beta\left(E_{i}+E_{j}\right) / 2\right)$ is small compared to the level spacing 
between $T_{i, i}$ and $T_{i+1, i+1}$. Since $E_{j}$ is uncorrelated from $E_{i}$, the matrix element $T_{i, j}$ must be averaged over $E_{j}$, so that $\tilde{T}_{i, j}$ is of the order of $\exp \left(\beta E_{i} / 2\right)$, that is

$$
\tilde{T}_{i, j} \sim \sqrt{\frac{\left|T_{i, i}\right|}{2}} .
$$

The distribution of eigenvalues of the unperturbed system is

$$
P(|T|)=\frac{1}{2^{1+1 / \beta}}|T|^{1 / \beta-1},
$$

from what we deduce the level spacing statistics in the long time (small $|T|$ ) approximation

$$
P_{|T|}(S) \simeq \frac{N}{\beta}(|T|+S)^{1 / \beta-1} .
$$

The off-diagonal terms do not affect the density of eigenvalues provided

$$
\int_{0}^{\sqrt{|T| / 2}} P(S) d S \ll 1,
$$

which leads to the condition $N^{2} \exp E \ll 1$. The average number of traps generated among a sequence of $N$ independent traps chosen among the distribution $P(E)=\exp E$ and fulfilling the aforementioned condition is

$$
N \int_{-\infty}^{-2 \ln N} e^{E} d E=\frac{1}{N}
$$

which is vanishing in the large $N$ limit. We thus conclude that the Arrhenius regime with this topology does not exist, that is off-diagonal terms will always modify significatively the density of eigenvalues with respect to the Arrhenius case. As we will see below, the Arrhenius regime is replaced by a renormalized Arrhenius regime, where the density of eigenvalues is given by (21), but with $\alpha$ lower than $1-T$.

\subsubsection{Numerical tests}

Again, we generate numerically the density of small eigenvalues $P_{B}(\ln |\lambda|)$. The fit is shown on figure 2, and the variations of the exponent in the Arrhenius regime are plotted on figure 3. We conclude to the existence of a renormalized Arrhenius regime, in the sense that the density of eigenvalues is given by (21) but with an exponent $\alpha$ smaller than $1-T$, indicating a slowing down of the dynamics due to the one dimensional topology compared to the infinite dimensional case: in one dimension, to go from one trap to a deeper trap, the system has to jump to intermediate traps.

\subsubsection{Relation with the anomalous diffusion on site-disordered lattices}

Section 2.2.3 was devoted to the connection between the spectrum of the Glauber matrix in infinite dimension and the long time behavior of random walks on finite $d$ dimensional trapped hypercubic 
lattices above the upper critical dimension. We now analyze the analogy in one dimension. We have shown numerically that the density of eigenvalues behaves like $\rho(\lambda) \sim \lambda^{1-T^{\prime}}$ for small $\lambda$, where $T^{\prime}$ is different from $T$. In terms of random walks, this result becomes, with the notations of section 2.2 .3

$$
P_{0}^{(R W)}(t) \sim t^{-T^{\prime} / 2}
$$

namely the spectral dimension is $d_{s}=T^{\prime}$. We now use the results of [18] where $d_{s}$ was calculated:

$$
T^{\prime}=d_{s}=2 \nu=\frac{2 T}{1+T}
$$

from what we conclude that the coefficient $\alpha$ plotted on figure 3 is

$$
\alpha=\frac{1-T}{1+T} .
$$

This analytic form is consistent with numerical calculations of the density of eigenvalues.

\subsection{One dimensional case with ordered traps}

We now assume that $N$ energy traps were generated among the distribution $P(E)=\exp E$, and that these energies have been ordered in such a way that $E_{1}<E_{2}<\ldots<E_{N}$. A site $k$ with a trap of energy $E_{k}$ is connected to the sites $k-1$ (with an energy $E_{k-1}$ ) and $k+1$ (with an energy $E_{k+1}$, except for the sites 1 and $N$ which have no neighbor. Intuitively, such a trap ordering should make the dynamics faster with respect to the case analyzed in section 2.3 since the system can decrease its energy continuously during the relaxation. We have

$$
\tilde{T}_{i, i+1}=\frac{1}{2} \sqrt{T_{i, i} T_{i+1, i+1}} \text {. }
$$

Since the density of level spacings $P_{|T|}(S)$ (31) is large for small values of $|T|$, we expect that $T_{i+1, i+1}$ is close to $T_{i, i}$ at least if $\left|T_{i, i}\right|$ is small, so that we can use the following approximation: $\tilde{T}_{i, i}=T_{i, i} / 2$. The off-diagonal terms will not modify the density of eigenvalues with respect to the Arrhenius case provided

$$
\int_{0}^{|T| / 2} P_{|T|}(S) d S \ll 1,
$$

that is $N \exp E \ll 1$. The number of traps fulfilling this condition in a distribution of $N$ traps is on average equal to unity, so that only a small number of eigenvalues contribute to the Arrhenius regime. Nevertheless, it is still possible to make a statistics over a large ensemble of $N$ traps distribution and to test numerically if the Arrhenius regime can be observed.

\subsubsection{Numerical tests}

We generated numerically the density of small eigenvalues $P_{B}(\ln |\lambda|)$. As shown on figure 2, we still can fit $P_{B}(\lambda)$ to the form $\exp (-\alpha \ln |\lambda|)$, and the exponent $\alpha$ is close to $1-T$, as in the Arrhenius regime. (see figure 3). We conclude that ordering the traps in one dimension restores the Arrhenius regime for the lowest eigenvalues. Again, the existence of the Arrhenius regime is sample dependent. 


\section{General relation between ageing and the Glauber matrix}

We are now going to give a general relation between ageing and the Glauber matrix. This relation is a generalization of a criterium given in [9]. In the context of the REM-like trap model, the average trapping time

$$
\langle\tau\rangle=\tau_{0} \int_{-\infty}^{0} d E \exp (E(1-\beta))
$$

diverges if $T<T_{g}=1$. On the other hand, ageing exists below $T_{g}$ only, in the limit where the microscopic time scale $\tau_{0}$ goes to zero. We are now going to argue that a similar criterium can be found for any statistical mechanical model, provided the master equation is linear. This is not always the case. For instance, the master equation of the Backgammon model in a mean field dynamics at zero temperature is not linear [16].

Let us now start our general argument. The idea is to start with a system in a given microcanonical state $\left|e_{i}\right\rangle$ and write the Glauber dynamics equation for the evolution of this pure state. First, we call $P$ the transformation matrix

$$
\left|e_{i}\right\rangle=\sum_{\alpha} P_{i, \alpha}\left|\Psi_{\alpha}\right\rangle
$$

where $\left|e_{i}\right\rangle$ is a microcanonical state and $\left\{\left|\Psi_{\alpha}\right\rangle\right\}$ are the eigenstates of the Glauber matrix. By microcanonical state, we mean that if the system is in the state $\left|e_{i}\right\rangle$, the system is with probability one in the microcanonical state $e_{i}$. In order to avoid confusion, all the labels related to the eigenvectors will be denoted by greek letters whereas the labels for pure states will be denoted by latin letters. The evolution of the pure state (40) is readily obtained as

$$
\left|e_{i}(t)\right\rangle=\sum_{\alpha, j} P_{i, \alpha} e^{\lambda_{\alpha} t} P_{\alpha, j}^{-1}
$$

In other words, the probability to be in microcanonical state $e_{j}$ at time $t$ starting from the microcanonical state $e_{i}$ is

$$
\mathcal{P}\left(e_{j}, t \mid e_{i} 0\right)=\sum_{\alpha} P_{i, \alpha} e^{\lambda_{\alpha} t} P_{\alpha, j}^{-1} .
$$

We now consider the time-dependent correlation function

$$
\mathcal{C}_{N}(t)=\frac{1}{\mathcal{N}} \sum_{i, j} \mathcal{P}\left(e_{j}, t \mid e_{i}, 0\right) q_{i, j},
$$

where $\mathcal{N}$ is the number of microcanonical states. We use $\mathcal{N}$ instead of $N$ to avoid confusion between the number of microstates $\mathcal{N}$ and the number of sites $N$ in a spin model. In a trap model, $\mathcal{N}=N$, whereas in an Ising spin system, $\mathcal{N}=2^{N}$. In (43), we have carried out an average over all the initial microcanonical states, that is the system is first quenched from an infinite temperature, and the correlation (43) is averaged over the realizations of the infinite temperature states. In (43), $q_{i, j}$ is the overlap between the pure states $e_{i}$ and $e_{j}$, which has to be defined within each model. As we 
will see below in section 1 , even within a given dynamics, the choice of the overlap depends on what type of observables are considered in the two times correlation functions. We now want to calculate from (43) a typical time by integrating (43). However, the Boltzmann eigenstate leads to a trivial divergence of this integral. We thus need a prescription to eliminate the equilibrium distribution in (43). We first discuss this prescription in the absence of metastability and postpone for later discussion the case of metastability. We define the typical time $\tau_{w}$ by

$$
\tau_{w}(N)=\frac{1}{\mathcal{N}} \overline{\sum_{i, j} \sum_{\alpha \neq \text { Bol. }} P_{i, \alpha} \frac{1}{\lambda_{\alpha}} P_{\alpha, j}^{-1} q_{i, j}},
$$

where the summation over the eigenstates excludes the Boltzmann distribution. The overline denotes an average over the disorder realizations, if necessary. The significance of $\tau_{w}(N)$ is clear: for a finite size system with $N$ sites, the out-of-equilibrium dynamics occurs before $\tau_{w}(N)$ and, for times larger than $\tau_{w}(N)$, the finite size system has equilibriated. In other words, $\tau_{w}(N)$ is the cross-over time before which the finite size system exhibits ageing. Notice that in this argument, the system is a finite size system. One should thus carefully study finite size effects. Two cases may occur: first, $\tau_{w}(N)$ tends to a constant in the thermodynamic limit. In this case, ageing is interrupted [0] in the thermodynamic limit. As we shall see, this is the case for the Ising chain at a finite temperature. On the other hand, if $\tau_{w}(N)$ diverges in the thermodynamic limit, ageing is not interrupted. In this case, the exitence of a divergent relaxation time with respect to times 0 and $t$ implies the existence of a divergent relaxation time with respect to any finite waiting time $t_{w}$ and $t+t_{w}$, and thus the existence of ageing in the thermodynamic limit. The relation between the existence/absence, the nature of ageing in the thermodynamic limit and the behavior of $\tau_{w}(N)$ was derived on heuristic basis. We could not find a rigourous derivation using a general argument. However, in what follows, we investigate the behavior of several models (REM-like trap model, random walks, BM model, one dimensional Ising chain and SK model) and we find in all these cases that our criterium based on the behavior of $\tau_{w}(N)$ is correct. As far as non interrupted ageing in the thermodynamic limit is concerned, we will see below that this is the case for the BM and SK models. This is also the case for the REM-like trap model. We have shown in section 2.2 that for all the typical realizations of the trap configurations (typical is defined in section 2.2), the density of eigenvalues is given by the Arrhenius law, which means that, in the case of the REM-like trap model, our criterium for the existence of ageing is equivalent to the criterium of Bouchaud [7] that we have recalled at the beginning of this section, provided the overlap between traps is taken to be $q_{i, j}=q_{E A} \delta_{i, j}$, where $q_{E A}$ is the Edwards-Anderson order parameter (within the one replica symmetry breaking step picture). Notice that the existence of a non Arrhenius regime generated by non typical trap configurations does not modify our conclusion regarding the divergence of $\tau_{w}^{R E M}(\infty)$ since the non Arrhenius regime only occurs for traps such as $E \leq-\ln N \rightarrow-\infty$ if $N \rightarrow \infty$. The correlation $\mathcal{C}_{\infty}^{R E M}(t)$ is

$$
\mathcal{C}_{\infty}^{R E M}(t)=\int_{-\infty}^{0} d E e^{E} \exp \left(-t e^{\beta E}\right)
$$


which is non integrable if $\beta \leq 1$. The typical time

$$
\tau_{w}^{R E M}(N)=-\frac{q_{E A}}{N} \overline{\sum_{\alpha \neq \text { Bol. }} \frac{1}{\lambda_{\alpha}}}=-q_{E A} \int_{-\infty}^{0} \frac{1}{\lambda} \rho_{N}(\lambda) d \lambda
$$

only depends on the density of eigenvalues $\rho_{N}(\lambda)$ for a system of $N$ traps, and is independent on the transition matrix, which motivates the study of the density of eigenvalues carried out in section

2. In general, in any model with only self-overlapping pure states, the existence of ageing is coded only in the behavior of the density of small eigenvalues: there is ageing in the thermodynamic limit if $\rho_{\infty}(\lambda)$ tends to a constant if $\lambda$ goes to zero (an exemple will be provided in section 5) or if $\rho_{\infty}(\lambda)$ diverges if $\lambda$ goes to zero (this is the case for the REM-like trap model already studied in section 2 ) .

Let us now discuss the behavior of the correlation function (43) in the presence of metastability. We have in mind the case of diamond for instance, which is metastable but in which case there is no ageing. We do not have a Glauber matrix description of diamond, but take this case as a generic situation for metastability and draw qualitative conclusions. In this case we expect that for a finite size system, (43) will first decay to a finite value over a first time scale $\tau_{w}^{(1)}(N)$. After $\tau_{w}^{(1)}(N)$, the system reaches its metastable configuration (for instance diamond) and thermalizes in a portion of its phase space. We thus expect that, for times larger than $\tau_{w}^{(1)}(N)$, the correlation (43) reaches a plateau. On larger time scales, the correlation (43) should decay to its equilibrium value with a cross-over time $\tau_{w}^{(2)}(N)$, corresponding for instance to the transition from diamond to graphite. The time scale $\tau_{w}^{(2)}(N)$ should increase with the number of sites, so that, with a macroscopic number of sites, no transition from diamond to graphite is observable on experimental time scales. On the other hand, $\tau_{w}^{(1)}(N)$ may remain small even in the thermodynamic limit, signaling the absence of ageing in the graphite phase. In such a situation where the cross-over time scale $\tau_{w}^{(2)}(N)$ is not observable, the only relevant time scale is $\tau_{w}^{(1)}(N)$. In order to calculate $\tau_{w}^{(1)}(N)$ one should not use the prescription (44) but rather substract to the correlation (43) its plateau value for $\tau_{w}^{(1)}(N) \ll t \ll \tau_{2}^{(2)}(N)$.

\section{Brownian motion}

Brownian motion was shown in [12] to be an ageing phenomenon. This model is simple and thus provides a simple test of the criterium of section 3. Brownian motion in an euclidian space of dimension $d$ has the following Glauber dynamics: $T_{i, i}=-1 / \tau_{0}$ and $T_{i, j}=1 /\left(2 d \tau_{0}\right)$ if $i$ and $j$ are nearest neighbors on a hypercubic lattice. $\tau_{0}$ is the microscopic hopping time. In this case, the Glauber matrix is symmetric and, because of translational invariance, its eigenstates are plane waves

$$
\left|\Psi_{\mathbf{k}}\right\rangle=\frac{1}{\sqrt{N}} \sum_{\mathbf{x}} e^{i \mathbf{k} \cdot \mathbf{x}}\left|\Psi_{\mathbf{x}}\right\rangle
$$


where $\mathbf{k}=\left(n_{1}, \ldots, n_{d}\right) 2 \pi / L$ (we have assumed cyclic boundary conditions with a lattice spacing $a$ ). The matrix elements of the Glauber matrix on the plane wave basis are

$$
\left\langle\Psi_{\mathbf{k}}|T| \Psi_{\mathbf{l}}\right\rangle=\delta_{\mathbf{k}, \mathbf{l}} \frac{1}{\tau_{0}}\left(-1+\frac{1}{d} \sum_{\alpha=1}^{d} \cos \left(k_{\alpha} a\right)\right),
$$

where $\alpha$ runs over the $d$ directions and $k_{\alpha}$ is the component of the wave vector $\mathbf{k}$ on the $\alpha$ direction. The long time behavior of the random walk is readily obtained by expanding the cosine up to the second order, and the probability to find the brownian particle at time $t_{w}$ on site $\mathbf{x}$ is, in the long time limit:

$$
\mathcal{P}\left(\mathbf{x}, t_{w} \mid \mathbf{0}, 0\right)=\frac{1}{(2 \pi)^{d}}\left(\frac{2 \pi d \tau_{0}}{t_{w}}\right)^{d / 2} \exp \left(-\left(\frac{d \tau_{0}}{2 t_{w} a^{2}} \mathbf{x}^{2}\right)\right)
$$

where the particle is at the origin at time $t=0$ (out of equilibrium state). As noticed first in [12], the two times correlation functions of the position are proportional to the waiting time:

$$
\mathcal{C}^{(1)}\left(t_{w}+t, t_{w}\right)=\left\langle\mathbf{x}\left(t_{w}+t\right) \cdot \mathbf{x}\left(t_{w}\right)\right\rangle=\left\langle\mathbf{x}^{2}\left(t_{w}\right)\right\rangle=\frac{t_{w} a^{2}}{d \tau_{0}}
$$

in the large $t_{w}$ limit. In order to make contact with [12], one should adopt the scaling $\tau_{0} \sim a^{2}$ in the thermodynamic/long time limits, which allows to recover the Langevin dynamics results and thus the existence of ageing in any dimension for the position-position correlation function.

In order to make contact between the correlation (50) and the general form of the correlation (43), we should choose the overlap $q(\mathbf{x})=\mathbf{x}^{2}$. With this choice of the overlap, (43) and (50) are both related to the same physical quantity: the mean square displacement. Using this specific form for the overlap, one can calculate $\mathcal{C}_{\infty}(t)$, integrate with respect to $t$ and show that $\tau_{w}(\infty)=\infty$, in agreement with the existence of ageing in this system for the position correlations.

One could also be interested in the following correlation:

$$
\mathcal{C}^{(2)}\left(t_{w}+t, t_{w}\right)=\left\langle\delta_{\mathbf{x}\left(t_{w}+t\right), \mathbf{0}} \delta_{\mathbf{x}\left(t_{w}\right), \mathbf{0}}\right\rangle=\mathcal{P}\left(0, t_{w} \mid 0,0\right) \mathcal{P}\left(0, t_{w}+t \mid 0, t_{w}\right) .
$$

In the large $t, t_{w}$ limit, this correlation is found to be

$$
\mathcal{C}^{(2)}\left(t_{w}+t, t_{w}\right)=\left(\frac{d \tau_{0}}{2 \pi}\right)^{d} \frac{1}{\left(t t_{w}\right)^{d / 2}} .
$$

In the thernodynamic limit, $a \rightarrow 0, \tau_{0} \sim a^{2}$, so that $\mathcal{C}^{(2)}$ is vanishing in the thermodynamic limit, signaling the absence of ageing for the correlation (51). The overlap associated to the correlation (51) is the local one: $q_{\mathbf{x}}=\delta_{\mathbf{x}, \mathbf{0}}$. We can thus calculate $\tau_{w}(\infty)$ using the integration (46) over the density of eigenvalues. In order to calculate the behavior of $\tau_{w}(N)$, we consider a finite system of volume $V=L^{d}$ with a lattice spacing $a$. The number $N$ of sites is $N=(L / a)^{d}$. By thermodynamic limit, we mean that $L$ is fixed, $a \rightarrow 0$ and $\tau_{0} \sim a^{2}$. Using (46), we have then

$$
\tau_{w}^{(2)}(N)=\left(\frac{a}{L}\right)^{d} \int \frac{d^{d} \mathbf{k}}{(2 \pi / L)^{d}} \frac{1}{\lambda_{\mathbf{k}}},
$$


where the summation is restricted over the wave vectors $\mathbf{k}$ such as $1 / L<\left|k_{\alpha}\right|<\pi / a$. Clearly, the dominant contribution to (53) comes from the smallest eigenvalues only. It is thus legitimate to expand the eigenvalues up to the quadratic order in $\mathbf{k}$ :

$$
\lambda_{\mathbf{k}} \simeq \frac{a^{2}}{\tau_{0} d} \mathbf{k}^{2}
$$

One then obtains

$$
\tau_{w}^{(2)}(N) \sim \frac{d \pi^{d / 2}}{2^{d-1} \Gamma(d / 2)} \tau_{0} a^{d-2} \int_{1 / L}^{\pi / a} k^{d-3} d k
$$

If $d=2$,

$$
\tau_{w}^{(2)}(N) \sim \pi \tau_{0}\left(\ln \left(\frac{\pi}{a}\right)-\ln \left(\frac{1}{L}\right)\right)
$$

Since $\tau_{0} \sim a^{2}, \tau_{w}^{(2)}(N) \rightarrow 0$ in the thermodynamic limit in two dimensions. Now if $d \neq 2$,

$$
\tau_{w}^{(2)}(N) \sim \tau_{0} \frac{d \pi^{d / 2}}{(d-2) 2^{d-1} \Gamma(d / 2)}\left(\pi^{d-2}-\left(\frac{a}{L}\right)^{d-2}\right) .
$$

Since $\tau_{0} \sim a^{2}$, this expression goes to zero in the thermodynamic limit whatever the dimension.

We have thus shown that, in the case of random walks, and in the case of the correlations (50) and (51), the existence/absence of ageing in the thermodynamic limit is consistent with the general argument of section 3 .

\section{The BM model at zero temperature}

\subsection{Diagonalization of the Glauber dynamics}

We assume that the traps have been ordered such as $E_{1}<E_{2}<\ldots<E_{N}$. Unlike the REM-like trap model, we do not make any asumption about the trap distribution. The finite temperature dynamics is

$$
T_{i, j}=\frac{1}{N} \frac{1}{1+\exp \left(\beta\left(E_{i}-E_{j}\right)\right)}
$$

if $i \neq j$ and satisfies detailed balance. The zero temperature form of the Glauber matrix of this model is very simple since $T_{i, i}=-i+1, T_{i, j}=0$ if $i>j$ and $T_{i, j}=1$ if $i<j$ at zero temperature. The spectrum is $\{0,-1 / N, \ldots,(-N+1) / N\}$. The eigenvector $\left|\psi_{0}\right\rangle$ for the eigenvalue $\lambda=0$ is $\left|e_{1}\right\rangle$. It is easy to show that the eigenvector $\left|\psi_{-k}\right\rangle$ associated to the eigenvalue $\lambda=-k / N$ is

$$
\left|\psi_{-k}\right\rangle=-\frac{1}{\sqrt{2}}\left|e_{k}\right\rangle+\frac{1}{\sqrt{2}}\left|e_{k+1}\right\rangle
$$

We can now express the pure states in terms of eigenvectors

$$
\begin{aligned}
& \left|e_{1}\right\rangle=\left|\psi_{0}\right\rangle \\
& \left|e_{k}\right\rangle=\left|\psi_{0}\right\rangle+\sqrt{2}\left(\left|\psi_{-1}\right\rangle+\ldots+\left|\psi_{-k+1}\right\rangle\right) .
\end{aligned}
$$


This means that, at zero temperature, a pure state $\left|e_{k}\right\rangle\left[k\right.$ is the $k$-ieth traps, with $\left.E_{1}<\ldots<E_{N}\right]$ overlaps only with eigenstates associated to eigenvalues $0,-1 / N, \ldots,(-k+1) / N$, and this overlap does not depend on the eigenstate. Compared to the result of section 2.2.4, the structure of the decomposition of a pure state into eigenstates of the Glauber dynamics is very different from the REM-like trap model.

If one starts from a pure state $\left|e_{k}(0)\right\rangle$ at time $t=0$, the state of the system at time $t$ is given by

$$
\left|e_{k}(t)\right\rangle=\left(1-e^{-t / N}\right)\left|e_{1}\right\rangle+\sum_{l=2}^{k-1}\left(e^{(-l+1) t / N}-e^{-l t / N}\right)\left|e_{l}\right\rangle+e^{(-k+1) t / N}\left|e_{k}\right\rangle .
$$

We recover the fact that this model can reach its ground state in a time of order $N$ at zero temperature, whereas in the limit of zero temperatures, and for the REM-like trap model, the time spent in a trap $i$ is proportional to $\exp -\beta E_{i}$, which goes to infinity if $\beta \rightarrow 0$ and $N$ fixed.

\subsection{Two times autocorrelation functions}

The two times autocorrelation functions were calculated in [15] by means of a Laplace transform formalism. The result is

$$
\Pi\left(t, t_{w}\right) \sim \frac{t_{w}}{t_{w}+t}
$$

if $1 \ll t, t_{w}$. We rederive this result in the Appendix using a treatment different from [15], and based only on the diagonalization of the Glauber matrix. The BM dynamics thus exhibits ageing.

\subsection{Relation to the general argument}

Provided only self overlaps are non zero $\left(q_{i, j}=\delta_{i, j}\right)$, the correlation function introduced in section 3 is $\mathcal{C}_{\infty}(t)=\frac{1}{t}$, provided $t \ll N$. The typical time $\tau_{w}^{B M}(\infty)$ below which ageing occurs is thus logarithmically divergent. On the other hand, this can be also shown directly from the spectrum:

$$
\tau_{w}^{B M}(N)=\sum_{i=1}^{N-1} \frac{1}{n}
$$

which diverges logarithmically.

\section{One dimensional Ising chain}

As far as the finite temperature behavior of the two times autocorrelation function is concerned, this model exhibits interrupted ageing [7] since at any finite temperature the correlation length is finite, of the order of $\xi=\exp (2 J / T)$, ageing does not exist after a time independent of the system size. It is thus interesting to apply to this case the general argument of section 3 . We first begin with the definition of the Glauber dynamics. 


\subsection{Glauber dynamics of the one-dimensional Ising chain}

We use the dynamics introduced in [13]. The master equation of this single spin flip dynamics is

$$
\frac{d}{d t} p(\{\sigma\}, t)=-\left(\sum_{i=1}^{N} w_{i}(\{\sigma\})\right) p(\{\sigma\}, t)+\sum_{i=1}^{N} w_{i}\left(\left\{\sigma_{1}, \ldots,-\sigma_{i}, \ldots, \sigma_{N}\right\}\right) p\left(\left\{\sigma_{1}, \ldots,-\sigma_{i}, . ., \sigma_{N}\right\}, t\right) .
$$

As a consequence of detailed balance,

$$
w_{i}(\{\sigma\})=\frac{1}{2}\left(1-\sigma_{i} \tanh \left(\beta J\left(\sigma_{i+1}+\sigma_{i-1}\right)\right) .\right.
$$

Notice that, unlike the case of the REM-like trap or BM models, there is no need to multiply the matrix elements by $1 / N$. It is shown in [21] that in the infinite temperature limit, the eigenvalues of the Glauber matrix are negative integers between $-N$ and 0 , with degeneracies given by the binomial coefficients. The largest relaxation time in the infinite temperature limit is equal to unity whatever the system size, and there is thus no need to rescale the coefficients of the Glauber matrix.

\subsection{Relation to the general argument}

In the case of a spin model, the overlap $q\left(\{\sigma\},\left\{\sigma^{\prime}\right\}\right)$ is given by

$$
q(\{\sigma\},\{\sigma ;\})=\frac{1}{N} \sum_{i=1}^{N} \sigma_{i} \sigma_{i}^{\prime}
$$

Since ageing is interrupted, we should observe a saturation of the time $\tau_{w}^{I \operatorname{sing} 1 D}(N)$ as $N$ increases. Moreover, $\tau_{w}^{I \operatorname{sing} 1 D}(N)$ should increase as the temperature decreases. In practise, we work with an open chain and we take advantage of the existence of the global $Z_{2}$ symmetry (invariance of the Glauber dynamics under the $\{\sigma\} \rightarrow\{-\sigma\}$ transformation in a zero magnetic field), and the reflection symmetry of the chain. The Glauber matrix is diagonalized in each of these four symmetry sectors. The results are plotted on figure 0 . The difference $\tau_{w}^{I \operatorname{sing} 1 D}(N)-\tau_{w}^{I \operatorname{sing} 1 D}(N-1)$ decreases as $N$ increases, in agreement with the existence of interrupted ageing.

\section{$7 \quad$ SK model}

This model was proposed in 1975 as an "exactly solvable" model of spin glasses [22]. For reviews on this model and its developments, we refer the reader to the reviews [23]. The Hamiltonian of the SK model is

$$
H=\sum_{\langle i, j\rangle} J_{i, j} \sigma_{i} \sigma_{j},
$$

where the sum is carried out over all the pairs of sites (infinite dimensional model) and the quenched random interactions are distributed according to a gaussian distribution

$$
P\left(J_{i, j}\right)=\left(\frac{N}{2 \pi}\right)^{1 / 2} \exp \left(-\frac{N J_{i, j}^{2}}{2}\right) .
$$


This model is a spin glass if $T<1$ and a paramagnet otherwise. The two times correlation function were shown in [5] to exhibit ageing in the glassy phase. We are going to apply our general argument

of section 3 to the case of the REM-like trap model. To do so, we calculate numerically $\tau_{w}^{S K}(N)$ for a large number of disorder realizations. The result is plotted on figure 6, as well as the differences $\Delta_{N}=\tau_{w}(N+1)-\tau_{w}(N)$ for $N=3,4,5,6$ sites. We observe that $\Delta_{N}$ increases at low temperatures and decreases at high temperature. The crossing point on the insert of figure 6 should be identified to the glass temperature. This temperature is not equal to unity since the system sizes are very small. Notice that even though the sizes are quite small, a large number of disorder configurations is required to have a significant statistics (of the order of 500000 independent disorder configurations). The computer time required was equivalent to 1000 hours of CPU time on a single processor. Our results are thus consistent with the existence of ageing in the low temperature phase, and no ageing in the high temperature phase.

\section{Conclusion}

We have thus carried out a detailed analysis of ageing from a Glauber dynamics point of view, with an emphasis on finite size effects. We have shown how to extract information from the Glauber matrix about ageing, and how to analyze finite size effects. In some simple cases with only self overlaps, the behavior of the time after which there is no ageing only involves the knowledge of the average density of eigenvalues $\rho_{N}(\lambda)$ when $\lambda \rightarrow 0^{-}$. This is the case for the REM-like trap model, in which case we have carried out a careful analysis of the density of small eigenvalues. Our analysis was confirmed by numerical diagonalizations of Glauber matrices of small clusters. In this case, we were able to related the typical behavior of the density of eigenvalues to the spectral dimension of the corresponding diffusion process.

Acknowledgements: R.M. acknowledges interesting discussions with A. Barrat, J.P. Bouchaud, S. Franz and N. Wingreen. The authors acknowledge B. Douçot for his interest to this work and his encouragements. R.M. also acknowledges the hospitality of NEC Research Institute at Princeton where part of this work was done.

\section{Appendix}

We are going to calculate the two times autocorrelation function $\Pi\left(t, t_{w}\right)$ using a method based only on the exact diagonalization of the Glauber matrix at a zero temperature. Starting from an infinite temperature state at time $t=0$,

$$
|\Psi(0)\rangle=\frac{1}{N} \sum_{i=1}^{N}\left|e_{i}\right\rangle,
$$


with $\left|e_{i}\right\rangle$ a pure state, the natural definition of the two times autocorrelation function within this model is

$$
\Pi\left(t, t_{w}\right)=\sum_{i=1}^{N} \mathcal{P}\left(i, t_{w}\right) \exp \Delta_{i} t
$$

where $\mathcal{P}\left(i, t_{w}\right)$ is the probability to find the system in the trap number $i$ at time $t_{w}$ and $\Delta_{i}$ is the escape rate from trap $i$, that is the diagonal element of the Glauber matrix $\Delta_{i}=T_{i, i}$. This definition of the waiting time dependent autocorrelation function is similar to the one used in [9]. Using the expression (59) of the eigenvectors and the decomposition (60) and (61) of the pure states into eigenvectors, we can calculate in a straightforward fashion the evolution of the system, starting from the state (70):

$$
\left|\Psi\left(t_{w}\right)\right\rangle=\left(1-\left(1-\frac{1}{N}\right) e^{-t_{w} / N}\right)\left|e_{1}\right\rangle+\frac{1}{N} \sum_{k=2}^{N} e^{-k t_{w} / N}\left[(N-k+1) e^{t_{w} / N}-(N-k)\right]\left|e_{k}\right\rangle .
$$

The probabilities $\mathcal{P}\left(i, t_{w}\right)$ are easily recovered: $\mathcal{P}\left(i, t_{w}\right)=\left\langle e_{i} \mid \Psi\left(t_{w}\right)\right\rangle$. After straightforward calculations, the dominant behavior of $\Pi\left(t, t_{w}\right)$ is obtained, in the limit $1 \ll t, t_{w} \ll N$ :

$$
\Pi\left(t, t_{w}\right) \sim \frac{t_{w}}{t+t_{w}}
$$

which is equivalent to the result of [15]. Notice that if $t_{w}$ is of the order of the number of traps, the system has reached equilibrium: the dynamics is stationary (the system is stuck in the lowest trap) and there is no ageing. 


\section{References}

[1] L. Lundgren, P. Svedlindh, P. Norblad and O. Beckman, Phys. Rev. Lett. 1ㅡ, 911 (1983); P. Norblad, L. Lundgren, P. Svedlindh and L. Sandlund, Phys. Rev. Lett. B $\underline{33}, 645$ (1988).

[2] M. Alba, M. Ocio, J. Hamman, Europhys. Lett. 2 45 (1986); J. Phys. Lett. 46 (1985) L-1101; M. Alba, J. Hamman, M. Ocio, P. Refregier, J. Appl. Phys. 61, 3683 (1987); E. Vincent, J. Hamman, M. Ocio, Recent Progress in Random Magnets, D.H. Ryan Ed. (World Scientific, Singapore, 1992).

[3] L. Lundgren, Journal de Physique Colloque C8 (1988), 1001 and references therein.

[4] D.S. Fisher, D.A. Huse, Phys. Rev. Lett. $\underline{56}, 1601$ (1986); Phys. Rev. B $\underline{38}, 373$ (1988); G.J. Koper, H.J. Hilhorst, Physica A $\underline{155}, 431$ (1989).

[5] L.F. Cugliandolo and J. Kurchan, J. Phys. A $\underline{27}, 5749$ (1994).

[6] S. Franz and M. Mézard, Europhys. Lett. 26, 209 (1994); Physica A $\underline{209}$, 48 (1994).

[7] J.P. Bouchaud, Jour. Phys. I France 2, 1705 (1992);

[8] J.P. Bouchaud, E. Vincent, J. Hamman, Jour. Phys. I France 4, 139 (1994);

[9] J.P. Bouchaud and D.S. Dean, Jour, Phys I France $\underline{5}, 265$ (1995).

[10] L.J. Cugliandolo, J. Kurchan, Phys. Rev. Lett. J. Phys. A 71173 (1993).

[11] C. Monthus and J.P. Bouchaud, J. Phys A $\underline{29}$ (1996) 3847.

[12] L.J. Cugliandolo, J. Kurchan and G. Parisi, J. Phys. I France $\underline{4}$ (1994), 1641.

[13] R.J. Glauber, Jour. Math. Phys. $\underline{4} 294$ (1963).

[14] B. Derrida, Phys. Rev. Lett. $\underline{45}$ (1980) 79; B. Derrida, Phys. Rev. B $\underline{24}, 2613$ (1981).

[15] A. Barrat and M. Mézard, Jour. Phys I (France), 모, 131 (1995).

[16] S. Franz and F. Ritort, J. Stat. Phys. $\underline{5}, 941$ (1996)

[17] B. Derrida and G. Toulouse, J. Physique Lett. $\underline{46}$ (1985) L223.

[18] J. Machta, J. Phys. A $\underline{18}$ (1985) L531.

[19] J. Machta, Phys. Rev. B $\underline{24}$ (1981) 5260; J .Stat. Phys. $\underline{30}$ (1983) 305.

[20] See for instance J.P. Bouchaud, A. Comtet, A. Georges and P. Le Doussal, J. Phys. (Paris) $\underline{48}$ (1987); J. Phys (Paris) $\underline{49}$ (1988) 369. 
[21] R. Mélin, Jour, Phys. I (France) $\underline{6}, 469$ (1996).

[22] D. Sherrington and S. Kirkpatrick, Phys. Rev. Lett. 35, 1792 (1975); S. Kirkpatrick and D. Sherrington, Phys. Rev. B $\underline{17}, 4384$ (1978)

[23] M. Mézard, G. Parisi and M. Virasoro, Spin glass theory and beyond, World Scientific Ed. (1993); K.H. Fisher and J.A. Hertz, Spin Glasses, Cambridge Studies in Magnetism (1991); V.S. Dotsenko, M.V. Feigelman and L.B. Ioffe, Spin Glasses and Related Problem, Physics Reviews, Hardwood Academic Publishers (1990). 


\section{Figure captions}

Figure 1:

Density of eigenvalues in the Arrhenius regime for the REM-like trap model and fit to the exponential form ( $\beta=9, N=75$ traps). The insert shows the same quantity, but with also the smallest eigenvalues. For these small eigenvalues, the Arrhenius picture is not valid.

Figure 2:

Density of small eigenvalues of the models of section 2, for $\beta=5$. (a): infinite dimensional case (REM-like trap model); (b): one dimensional case, with "disordered" traps; (c): one dimensional case with "ordered" traps. The lines in cases (b) and (c) are parallel, and in good agreement with the Arrhenius law, whereas we observe a significant deviation to the Arrhenius law in (b) case.

Figure 3:

Exponent $\alpha$ deduced from the fit (21) of $P_{B}(\ln |\lambda|)$. The circles correspond to the infinite dimensional case (REM-like trap model), the squares to the one dimensional "disordered" case, and the diamonds to the "ordered" one dimensional case. The solid line corresponds the the Arrhenius regime (in other words, to site-disordered random walks above the upper critical dimension 2), and the dashed line corresponds to the one dimensional renormalized Arrhenius regime (in other words, to site-disordered random walks in one dimension).

Figure 4:

Structure of the eigenstates of the REM-like trap model for 20 traps. The label $i$ is an eigenvalue label, with eigenvalues in growing order and $j$ labels the traps (the depth increases if $j$ increases). (a) represents $\left\langle\psi_{i} \mid e_{j}\right\rangle \mid$ for the 20 pure states. (b) represents the depth of the traps. (c) represents the 20 eigenvalues between -1 and 0 for 4 different temperatures (circles: $\beta=2$, squares: $\beta=5$, diamonds: $\beta=10$, triangles: $\beta=20$ ).

Figure 5:

Variations of $\tau_{w}^{I \operatorname{sing} 1 D}(N)$ as a function of the inverse temperature $\beta$ for $N=3,4,5,6,7,8,9$ sites. The insert shows the differences $\tau_{w}^{I \operatorname{sing} 1 D}(N)-\tau_{w}^{I \operatorname{sing} 1 D}(N-1)$ for $N=4,5,6,7,8,9$ sites (curves a,b,c,d,e,f respectively).

Figure 6:

Variations of $\tau_{w}^{S K}(N)$ as a function of the inverse temperature $\beta$ for a $N=3,4,5,6 \mathrm{SK}$ cluster. The insert shows $\tau_{w}^{S K}(N)-\tau_{w}^{S K}(N-1)$ [a: $\left.N=6, \mathrm{~b}: N=5 \mathrm{c}: N=4\right]$. 


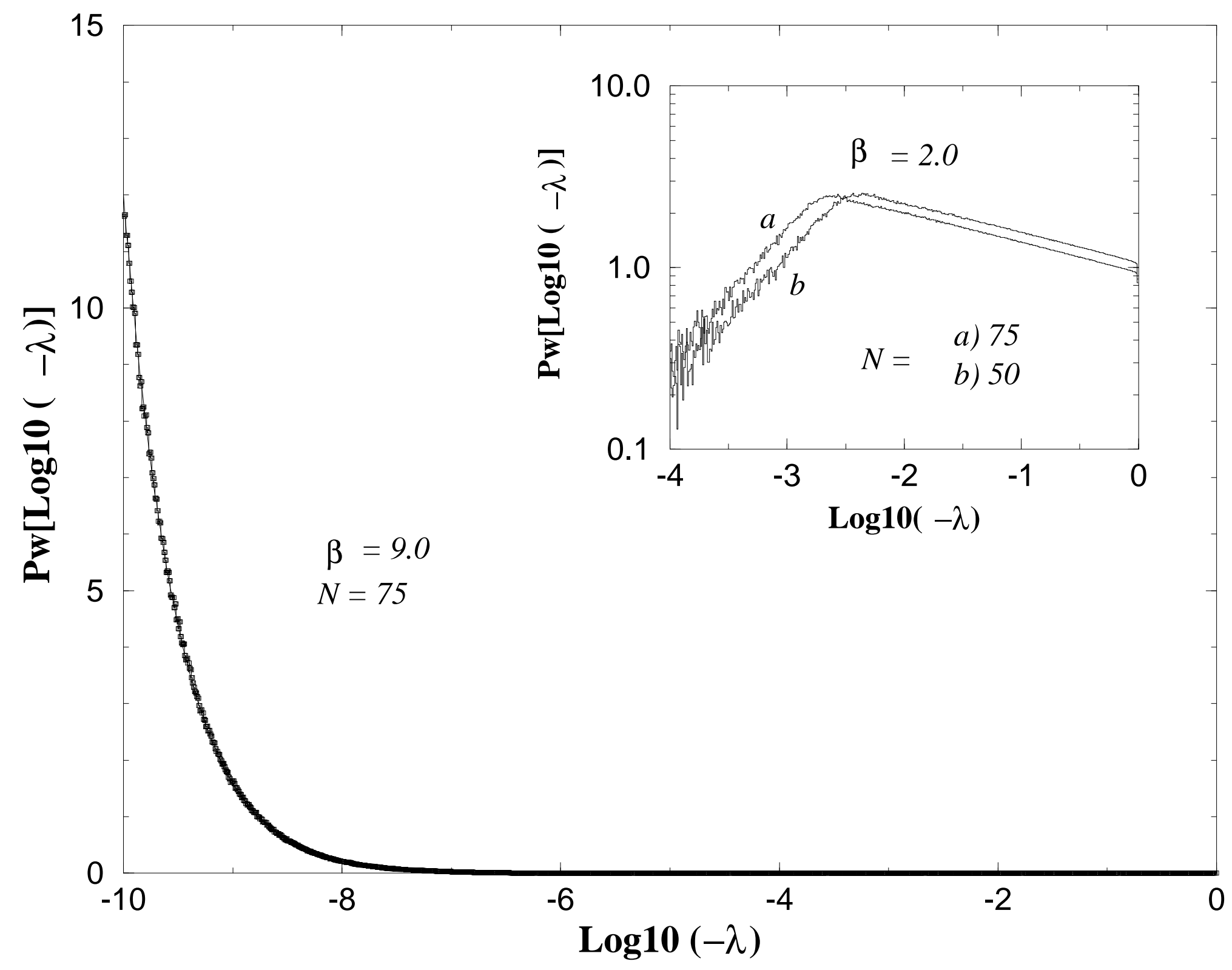




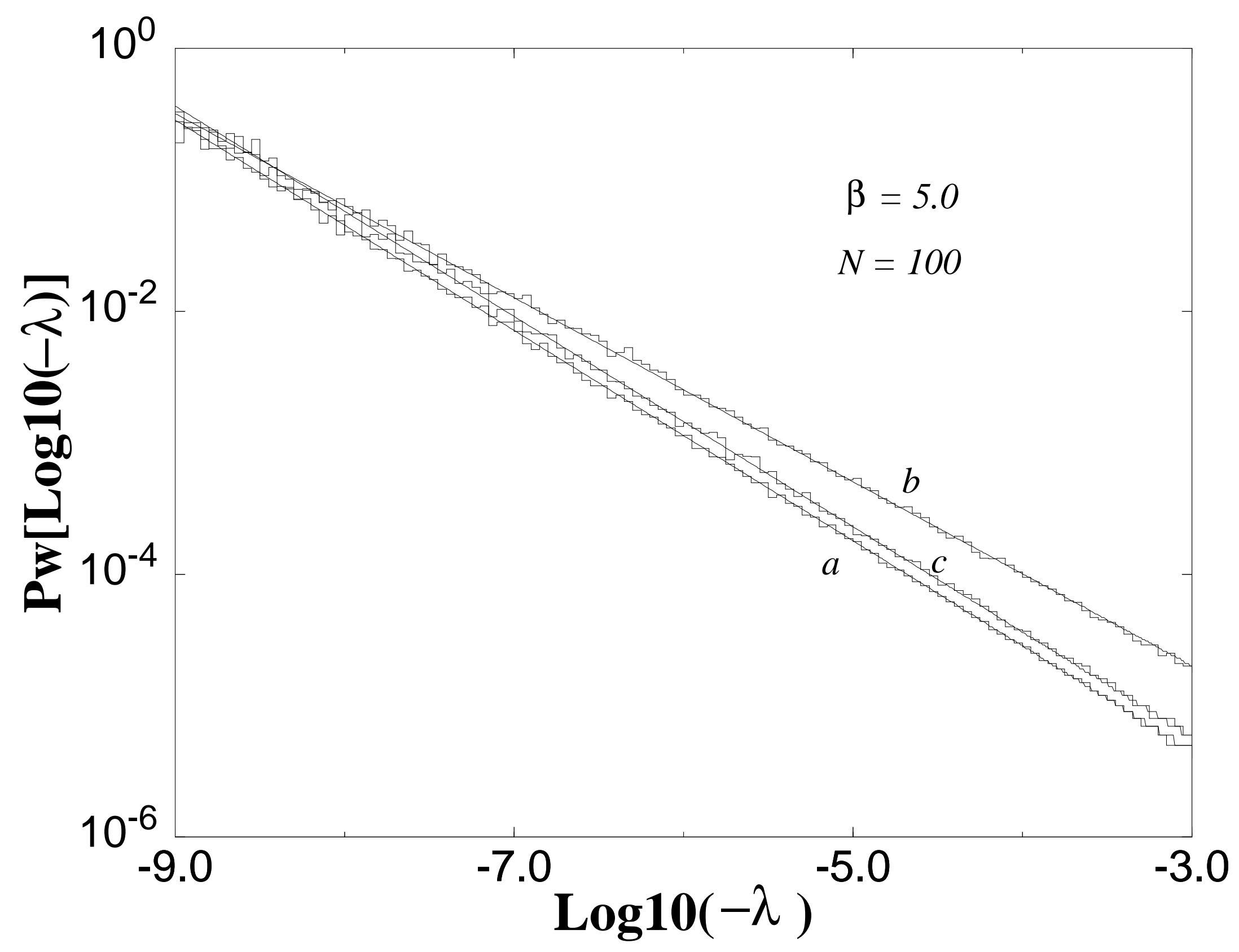




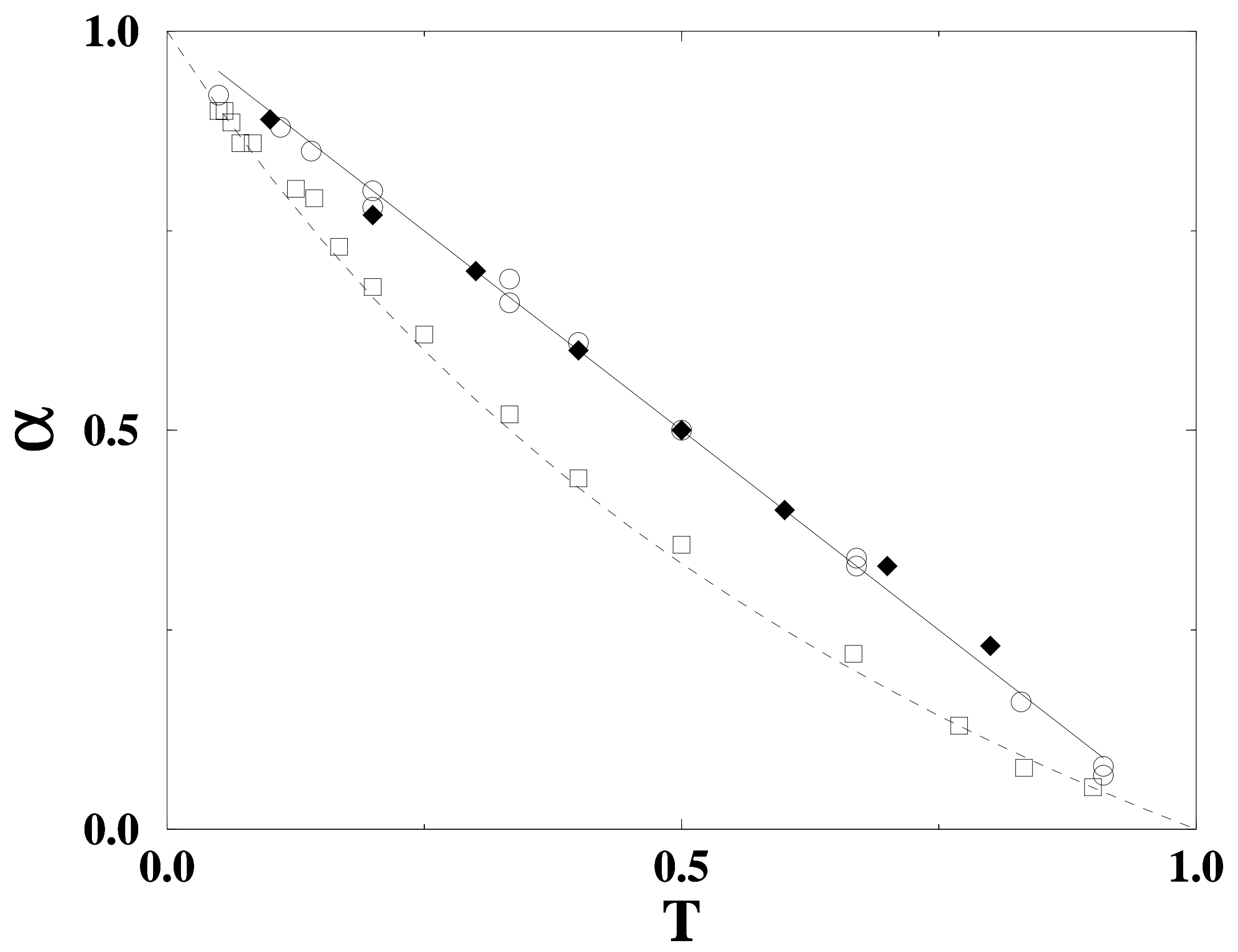




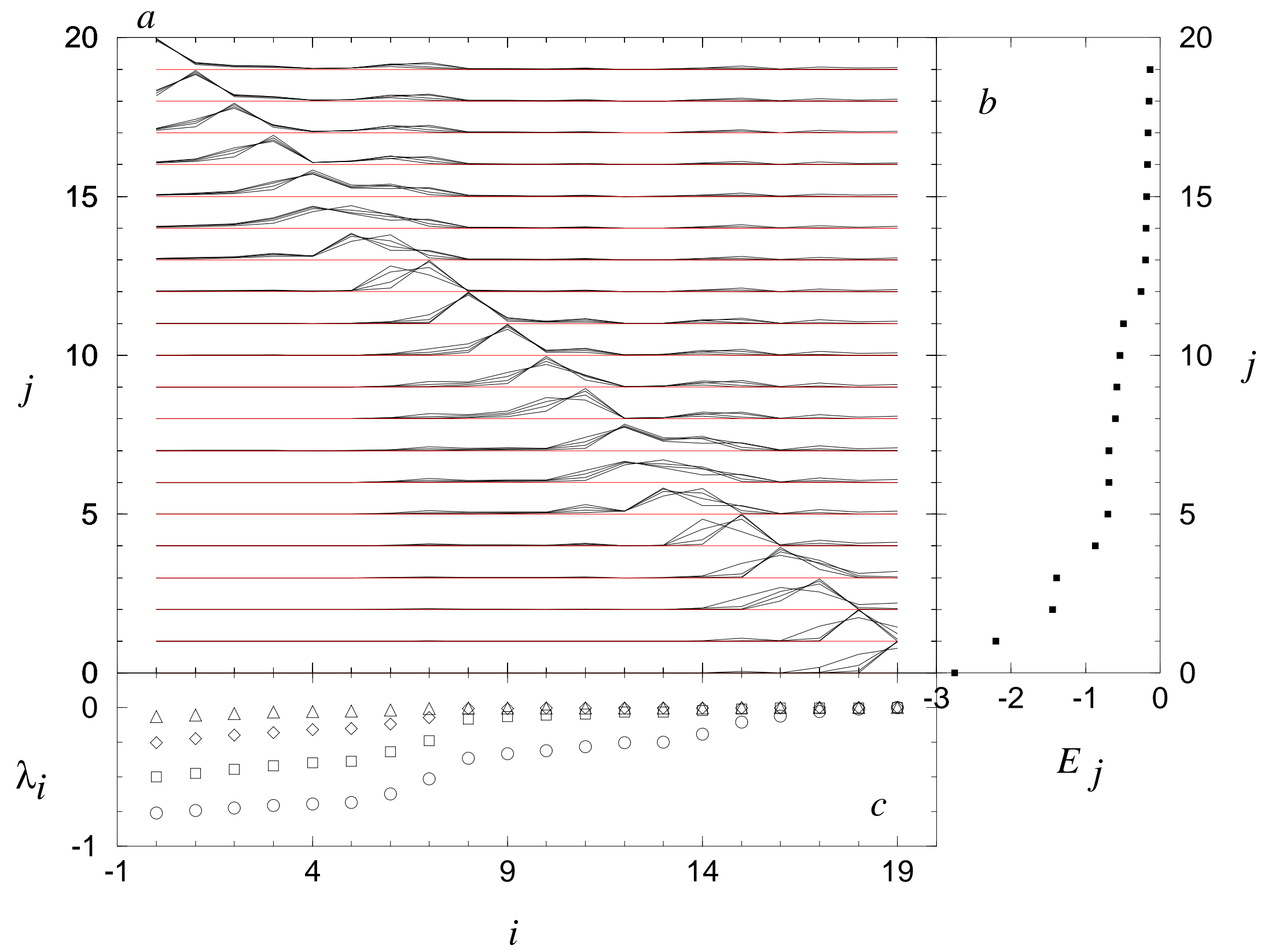




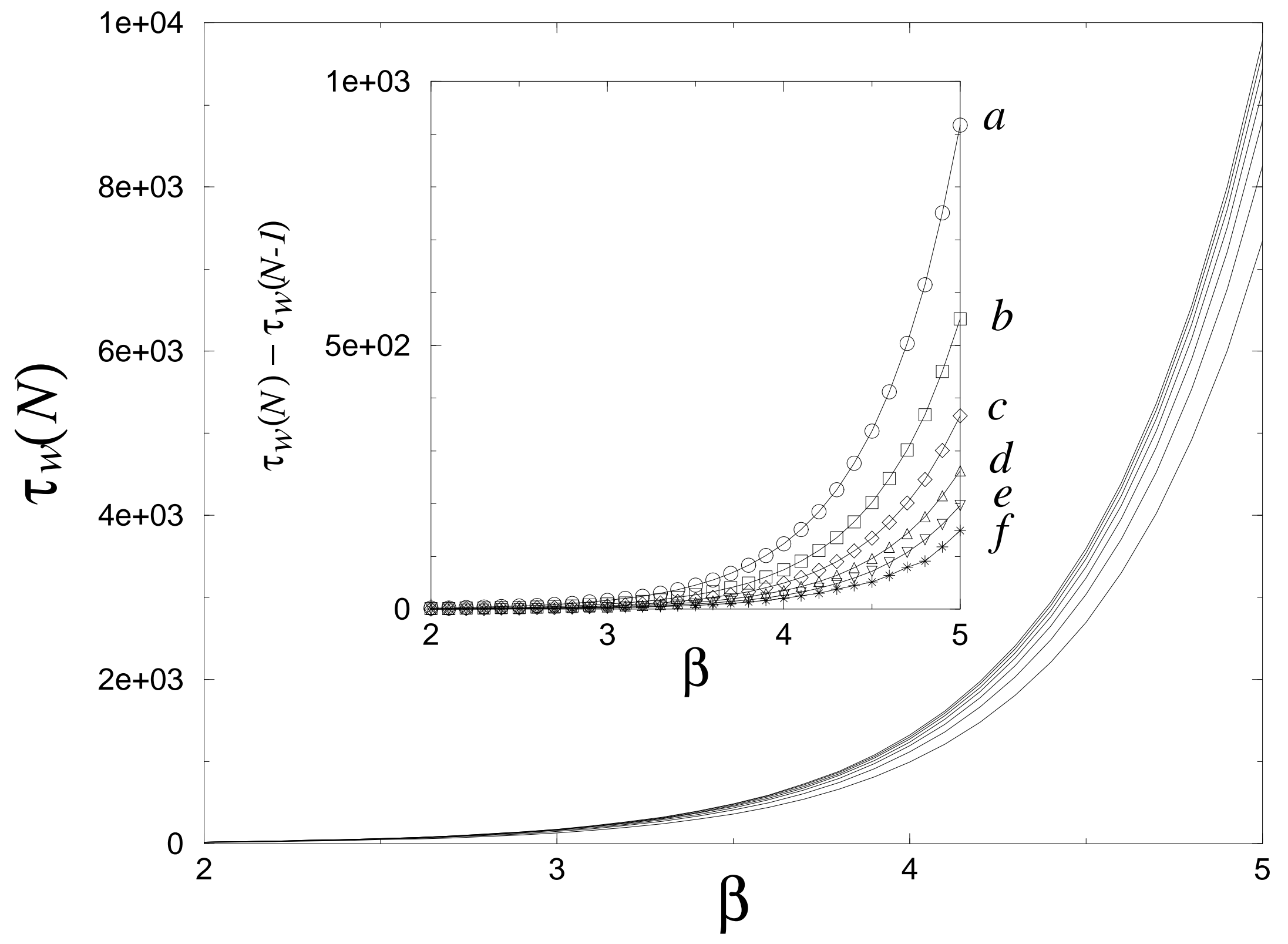




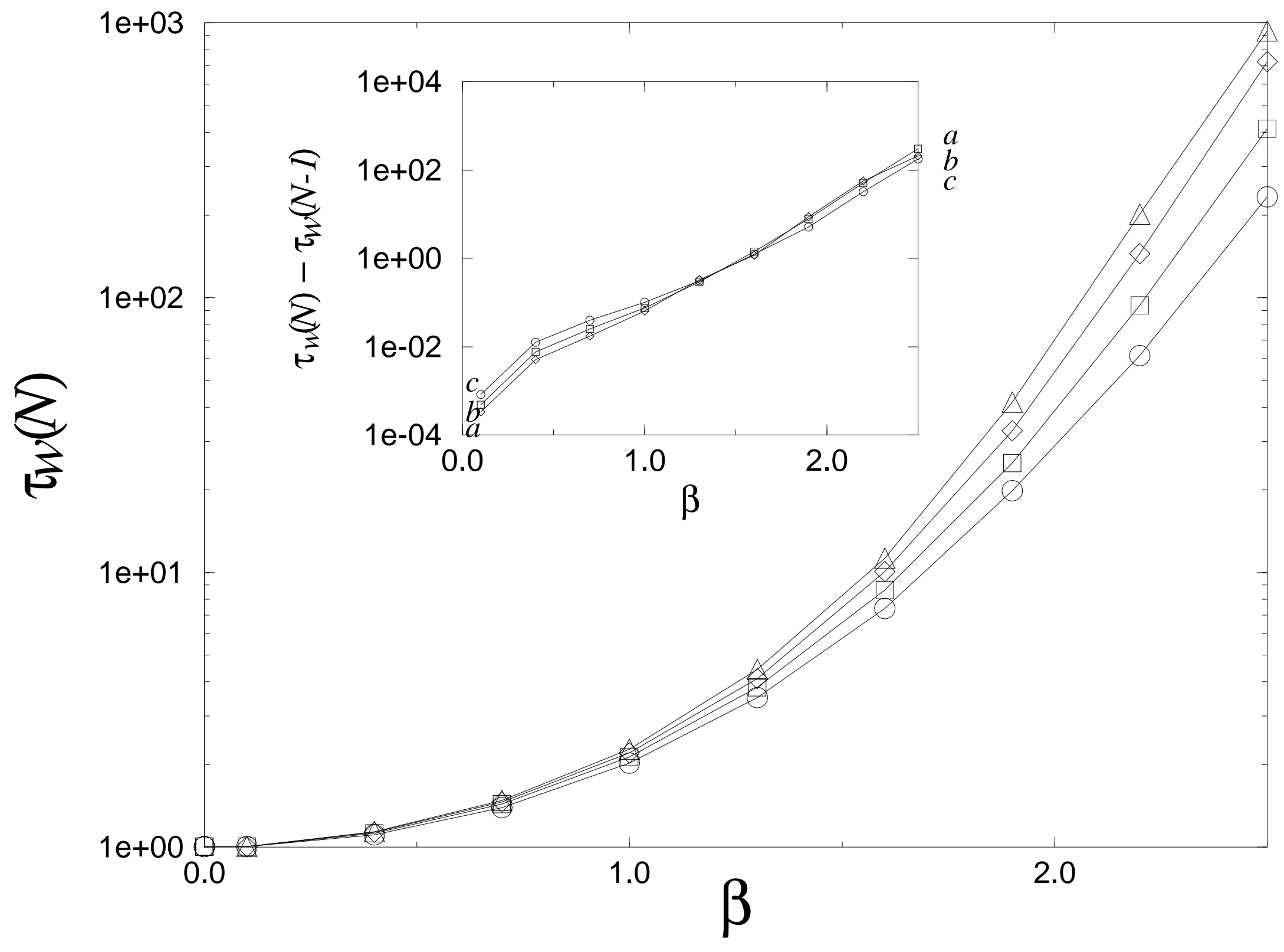

